\title{
How to Control Controlled School Choice
}

\author{
By Federico Echenique And M. Bumin Yenmez*
}

\begin{abstract}
We characterize choice rules for schools that regard students as substitutes while expressing preferences for a diverse student body. The stable (or fair) assignment of students to schools requires the latter to regard the former as substitutes. Such a requirement is in conflict with the reality of schools' preferences for diversity. We show that the conflict can be useful, in the sense that certain unique rules emerge from imposing both considerations. We also provide welfare comparisons for students when different choice rules are employed. (JEL D47, H75, I21, I28)
\end{abstract}

Recent school choice programs seek to install a stable (or "fair," to use the terminology of school choice) assignment of students to schools (Abdulkadiroğlu and Sönmez 2003; Abdulkadiroğlu et al. 2005). This objective is severely compromised by school districts' concern for diversity. Under diversity considerations, a stable assignment may not exist, and the mechanisms used in reformed school districts may not work.

There is a very basic tension between diversity considerations and the requirements of stable matching: diversity considerations introduce complementarities in schools' choices; but the theory of stable matchings requires substitutability. If a school is concerned with gender balance, for example, then it may admit a mediocre male applicant only to maintain gender balance because it has admitted an excellent female applicant. The female applicant cannot be admitted without the male applicant: the two students are thus complements, not substitutes, for the school. Complementarities in the school's choices of students are a problem because both the theory and the mechanism proposed in school choice programs require that students be substitutes in schools' choices. We are far from the first to recognize this problem: see a discussion of relevant literature below. The idea that diversity clashes

\footnotetext{
*Echenique: Division of the Humanities and Social Sciences, California Institute of Technology, 1200 E. California Boulevard, Mail Code 228-77, Pasadena, CA 91125 (e-mail: fede@caltech.edu); Yenmez: Tepper School of Business, Carnegie Mellon University, 5000 Forbes Avenue, Pittsburgh, PA 15213 (e-mail: byenmez@ andrew.cmu.edu). We thank Estelle Cantillon, Lars Ehlers, Sean Horan, Mark Johnson, Juan Pereyra Barreiro, Ariel Rubinstein, Kota Saito, Yves Sprumont, and Utku Ünver for their helpful comments, as well as seminar audiences at multiple universities and conferences. We are particularly grateful to Scott Kominers, Parag Pathak, and Tayfun Sönmez for their comments and advice on existing controlled school choice programs. Karen Hannsberry, manager at the office of access and enrollment at Chicago Public Schools, very kindly answered all our questions about their program. Finally, we are very grateful to the three anonymous referees for their suggestions, which have led to a much improved version of the paper. Yenmez gratefully acknowledges financial support from National Science Foundation grant SES-1326584. The authors declare that they have no relevant material or financial interests that relate to the research described in this paper.

$\dagger$ Go to http://dx.doi.org/10.1257/aer.20130929 to visit the article page for additional materials and author disclosure statement(s).
} 
with stability is very easy to recognize; in Section IA we present a particularly simple example of the incompatibility between stability and diversity concerns.

Our paper seeks to reconcile diversity with the objective of implementing a stable matching of students to schools. We characterize the schools' choices that are compatible with both diversity considerations and the theory of stable matchings. There is so much tension between substitutability and diversity that one might think no choice rule can satisfy both. We show that this need not be the case: we study the choices that satisfy certain normative axioms, one of them being substitutability, and show how combinations of axioms give rise to unique choice procedures. Our procedures allow schools to express concerns for diversity while using the standard mechanism (the one used in the school choice programs guided by stable matching theory) to install a stable assignment of students to schools. ${ }^{1}$

We assume that students belong to one of multiple types. Types could be categories of gender, socioeconomic status, race, or ethnicity. In all our results, an "ideal" or "target" distribution plays a crucial role. Discussions over diversity in school choice often express the idea that each school should have a distribution of white, black, Hispanic, etc. children that matches, as closely as possible, the distribution of races and ethnicities in the relevant population. This idea is present in the literature on education (e.g., Alves and Willie 1987), as well as in actual school districts' criteria for affirmative action (see Fiske 2002).

Broadly speaking, our paper uses ideas and models from choice theory (individual, or social choice) to obtain results that we believe are useful for practical market design. We present three results. Each one says that a unique school choice rule emerges from some combination of policy desiderata, expressed as axioms. We imagine that a school district can discuss a menu of axioms and will settle on the axioms that it deems most desirable. The school choice reforms in Boston and New York were based on offering school administrators a choice between stability and efficiency (Abdulkadiroğlu et al. 2005). We envision using our results by expanding the menu of axioms to include axioms that deal with diversity.

Our first result is to axiomatize a rule that tries to minimize the distance between the distribution over types in the student body and a given ideal distribution over types (see Section IIA). This rule thus operationalizes the criterion mentioned above, in which districts (or schools) target a particular distribution of types. The axiomatization tells us what such a rule means in terms of normative qualitative axioms.

In two other rules, the school sets aside a number of seats for each type of student (quotas and reserves; see Section IIB). The number of seats set aside for each type is related to the target distribution over types.

In our model, schools have two sources of preferences. They have given "priorities," which are preferences over individual students. These priorities can result from test scores, from the distance of the student's residence to the school, or some other objective criteria. The school also has preferences over the composition of the student body: these preferences come from concerns about diversity. The school or the district may combine these two preferences in different ways. Our results give

\footnotetext{
${ }^{1}$ We do not propose any new mechanisms: we want a theory that will work with the mechanisms that have already been accepted and adopted by multiple school districts. Indeed, these mechanisms have been accepted across many different market design problems (Roth 2008), such as markets for entry-level professional jobs.
} 
recommendations on how the combination should be carried out. If a school or a district agrees on a set of axioms, then there will be a unique way of combining priorities and diversity preferences into a choice rule for the school. As we shall see, substitutability, imposed as an axiom, has very strong implications for how to combine priorities and preferences over the composition of the student body.

Our analysis takes the priorities over individuals as given, which is in line with practice. Therefore, some of our axioms depend on an exogenously specified priority over students, and their validity may be a function of those priorities. In the online Appendix, we show that our analysis can also be carried out with endogenous priorities.

Finally, one might envision achieving diversity by directly manipulating priorities. For example, underrepresented groups may be given a high priority. This possibility falls outside of the model with exogenous priorities; but we feel that the admissions rules (ideal points, reserves, and quotas) with exogenous, non-diversity-related priorities are important enough in the debate on school choice to warrant the focus on exogenous priorities.

Related Literature.-Abdulkadiroğlu and Sönmez (2003) introduced matching theory as a tool in school choice and noted the problem with diversity concerns; they offer a solution based on quotas, one of the models we axiomatize below. 2

The last two years have seen multiple explorations into controlled school choice and diversity concerns. Kojima (2012) shows that affirmative action policies based on majority quotas may hurt minority students. To overcome this difficulty, Hafalir, Yenmez, and Yildirim (2013) propose affirmative action based on minority reserves. More generally, Ehlers et al. (2014) study affirmative action policies when there are both upper and lower type-specific bounds. They propose solutions based on whether the bounds are hard or soft. In contrast, our paper seeks to endogenize the rules and consider (possibly) all of them. Other papers consider specific choice rules, including some that are close to our reserves rule (Aygün and Bo 2013; Westkamp 2013; Kominers and Sönmez 2012).

In contrast with other papers in the literature, our focus is not on the market as a whole, but rather on the preferences or choices of individual schools. However, we consider the welfare implications of different choice rules on students in Section III using a general comparative static result that we provide in the online Appendix.

We focus on school preferences, but student preferences may also induce problems: for example, students may have preferences over their colleagues. These problems are treated by Echenique and Yenmez (2007) and Pycia (2012); they are outside the scope of the present analysis. We focus here on diversity and its effects on standard stable matching theory.

${ }^{2}$ See Kahlenberg (2002) for controlled school choice in practice. 


\section{Model}

Our model has three components: a choice rule, which describes the admissions policy of the school; a priority order, which ranks individual students according to how desirable they are to the school; and a partition of students into "types."

Let $\mathcal{S}$ be a nonempty finite set of all students. A choice rule is a function $C$ that maps each nonempty set $S \subseteq \mathcal{S}$ to a subset $C(S) \subseteq S$ of chosen students. Choice rules are both a basic tool in microeconomic theory, and a common modeling device in matching theory (for example Alkan and Gale 2003; Hatfield and Milgrom 2005). The interpretation of $C$ is that, if a school had the ability to admit its students out of the set $S$ of students, then it would choose $C(S)$ to be its student body.

We shall assume that there is a positive number $q$ such that $|C(S)| \leq q$ for all $S \subseteq \mathcal{S}$. The number $q$ is the capacity of the school: the number of available seats that it has.

A priority, or a (strict) preference, on $\mathcal{S}$ is a binary relation $\succ$ on $\mathcal{S}$ that is complete, transitive, and antisymmetric.

The set of students $\mathcal{S}$ is partitioned into students of different "types," which can be based on gender, socioeconomic factors, race, or ethnicity. Formally, there exists a set $T \equiv\left\{t_{1}, \ldots, t_{d}\right\}$ of types, and a type function $\tau: \mathcal{S} \rightarrow T$, where $\tau(s)$ is the type of student $s$. Let $\mathcal{S}^{t}$ be the set of type-t $t$ students; i.e., $\mathcal{S}^{t} \equiv\{s \in \mathcal{S}: \tau(s)=t\}$. Similarly, for any set of students $S \subseteq \mathcal{S}, S^{t}$ denotes the subset of $S$ that includes all type $t$ students; i.e, $S^{t} \equiv S \cap \mathcal{S}^{t}$.

We use a function $\xi: 2^{\mathcal{S}} \rightarrow \mathbf{Z}_{+}^{d}$ to describe the number of students of each type in each particular set. So we let

$$
\xi(S) \equiv\left(\left|S^{t_{1}}\right|, \ldots,\left|S^{t_{d}}\right|\right) \in \mathbf{Z}_{+}^{d},
$$

which consists of the number of students of each type in $S$. We term $\xi(S)$ the distribution of students in $S$.

We assume that the school is not large enough to admit all students of a given type: $q<\left|\mathcal{S}^{t}\right|$ for all $t \in T$.

\section{A. Gross Substitutes}

Our paper deals with the conflict between stability and diversity considerations. At the root of the conflict lies the property of gross substitutes. We first present a very stylized example illustrating the problem.

Suppose that there are two schools, $c_{1}$ and $c_{2}$, and two students, $s_{1}$ and $s_{2}$. The students are of different types. For example, $s_{1}$ and $s_{2}$ could be of a different race or ethnicity.

School $c_{1}$ can admit two students, but it is constrained to mimic the population representation of each type. So it must admit either both students or neither. School $c_{2}$ has a single empty seat. It prefers to admit student $s_{1}$ over student $s_{2}$, meaning that student $s_{1}$ has higher priority than $s_{2}$ in school $c_{2}$.

Students have preferences over schools: $s_{1}$ prefers $c_{1}$ over $c_{2}$, while $s_{2}$ 's favorite school is $c_{2}$. The table below summarizes all agents' preferences. 


\begin{tabular}{cc|cc}
$c_{1}$ & $c_{2}$ & $s_{1}$ & $s_{2}$ \\
\hline$\left\{s_{1}, s_{2}\right\}$ & $s_{1}$ & $c_{1}$ & $c_{2}$ \\
& $s_{2}$ & $c_{2}$ & $c_{1}$ \\
\hline
\end{tabular}

It is easy to see that no matching of students to schools is stable. For example, if both students are assigned to school $c_{1}$, then $s_{2}$ might request the empty slot in school $c_{2}$. School $c_{2}$ finds $s_{2}$ acceptable, so the pair $\left(c_{2}, s_{2}\right)$ can "block" this assignment (equivalently, $s_{2}$ 's claim to the empty seat is "justified," using the standard terminology in school choice; see Abdulkadiroğlu and Sönmez 2003). Similarly, if $s_{2}$ is assigned to $c_{2}$ then $s_{1}$ would have no place, as school $c_{1}$ cannot admit an unbalanced student body. Then $s_{1}$ would claim $s_{2}$ 's spot in school $c_{2}$. Since $s_{1}$ has a higher priority than $s_{2}$ at that school, $\left(c_{2}, s_{1}\right)$ can "block" this assignment (equivalently, $s_{1}$ "justifiably envies" $s_{2}$ in $c_{2}$ ). Thus the assignment of $s_{2}$ to $c_{2}$ is unstable. Finally, if $s_{1}$ is assigned to school $c_{2}$ and $s_{2}$ is unassigned, then both students would prefer school $c_{1}$, and school $c_{1}$ prefers both of them. Therefore, $\left(c_{1},\left\{s_{1}, s_{2}\right\}\right)$ can block the assignment.

There is no stable or fair assignment of students to schools in this example. The reason is that $c_{1}$ 's preferences for diversity cause the students $s_{1}$ and $s_{2}$ to be complements. Complementarities in the school's preference make it impossible to have a stable assignment.

The axiom needed to avoid such problems is gross substitutes.

GROSS SUBSTITUTES (GS): If a student is chosen from set $S$, then the student is also chosen from any subset of $S$ that contains her.

GS says that no student should be chosen because he or she complements another student. Consider the choices of school $c_{1}$ in the example. The school would accept $s_{1}$ if both students had applied, but it would reject $s_{1}$ if only $s_{1}$ had applied for its seats. Therefore, $c_{1}$ 's choice rule does not satisfy GS.

If all schools' choices satisfy GS, then a stable matching exists and the mechanism proposed by the recent school choice literature works well. GS was first studied by Kelso and Crawford (1982). GS is sufficient for the existence of stable matchings and for the Gale-Shapley deferred acceptance algorithm to find a stable matching. It is also in a sense necessary for these properties to hold (Hatfield and Milgrom 2005).

\section{B. Gross Substitutes as a Stand-Alone Normative Consideration}

We have argued that GS is appealing because it enables the use of the most popular and successful mechanism in school choice. There is another argument for GS. We claim that GS may be desirable even when admissions are not governed by a centralized mechanism.

Consider a school that processes applications in batches, making decisions sequentially. GS guarantees that a school will never decide to go back on a rejection. This is a desirable property of the school's choice rule. Consider, for example, 
the admissions procedure to a graduate program. In a first stage, a department may want to turn down certain students, admit others, and wait-list others. To turn down a student is a decision that is hard to back down from. GS says that the admissions committee will never need to reconsider a decision to reject a student.

\section{Results}

We characterize rules that differ in how they trade off concerns for diversity with concerns for the quality, or priority, of individual students.

Our first rule puts diversity first. It is called the ideal point rule, because it seeks to achieve a distribution over student types that is as close as possible to some given ideal distribution. Once the distribution over types is fixed, the rule admits the highest-priority students of each type. The diversity-first ideal point rule will never trade off a low-priority student for a high-priority student when they are of different types.

Then we turn to rules with flexible diversity, in which we sometimes allow priorities to guide the choice over students of different types. Of course, if we let priorities always guide choices, then the school may completely miss its diversity objectives. So we need to say precisely when the priority will be allowed to guide choice.

The key idea is that the number of students of a type tells us when priorities are allowed to guide choices. In one axiom, we require that once we have enough students of one type (where the notion of enough depends on how one treats students in other situations), then the admission of a student of that type cannot be due to diversity considerations - it must be because of priorities. In another axiom we take the opposite route, and say that the rejection of a student cannot be due to diversity when we do not have enough of that student's type.

Each of these two axioms leads to a rule of its own. In the first case, it leads to a rule that achieves diversity by reserving seats for different student types. In the second, it leads to a rule that achieves diversity by capping the number of students that the school can admit of each type.

\section{A. Diversity First}

We describe a rule that emphasizes diversity over individual students' priorities. It first decides on a distribution over types, and then admits the highest-priority students of each type.

The first axiom, monotonicity, is responsible for putting diversity first. Monotonicity says that having more students of each type available leads to more students (or at least as many) being admitted of each type-regardless of priorities.

MONOTONICITY: When $S^{\prime}$ has at least as many students of each type as $S$, then $C\left(S^{\prime}\right)$ has at least as many students of each type as $C(S)$.

One implication of monotonicity is that whenever two sets have the same number of students for each type, then the same number of students must be chosen from both sets for each type. This implication captures the idea of putting diversity above priorities. 
The second axiom simply says that, when comparing students of the same type, the choice should be guided by the students' priorities.

WITHIN-TYPE $\succ$-COMPATIBILITY: When a student is chosen over another student and they both have the same type, then the chosen student must have higher priority than the non-chosen student.

A choice rule is generated by an ideal point for priority $\succ$ if there exists an ideal distribution of students $\mathbf{z}^{*}$ such that the choice rule first chooses a distribution of students that is as close to $\mathbf{z}^{*}$ as possible, and then admits the best students of each type according to priority order $\succ$.

More formally, a choice rule $C$ is generated by an ideal point for priority $\succ$ if there exists a vector $\mathbf{z}^{*} \in \mathbf{Z}_{+}^{d}$ with $\left\|\mathbf{z}^{*}\right\| \leq q$ such that for any $S \subseteq \mathcal{S},{ }^{3}(\mathrm{i}) \xi(C(S))$ is the closest vector to $\mathbf{z}^{*}$ (in Euclidean distance), ${ }^{4}$ among those in $B(\xi(S)$ ), where

$$
B(\mathbf{x}) \equiv\left\{\mathbf{y} \in \mathbf{Z}_{+}^{d}: \mathbf{y} \leq \mathbf{x} \text { and }\|\mathbf{x}\| \leq q\right\}
$$

and (ii) type- $t$ students in $C(S)$ have higher priority than any type- $t$ student in $S \backslash C(S)$, for any $t$.

Our first result is a characterization of ideal point rules.

THEOREM 1: A choice rule is generated by an ideal point for priority $\succ$ if and only if it satisfies gross substitutes, monotonicity, and within-type $\succ$-compatibility.

The proof is in the Appendix at the end of the paper. In the online Appendix we also verify the independence of the axioms used in all three theorems.

\section{B. Flexible Diversity}

We now turn to rules that are flexible in how they incorporate diversity objectives. A school can now trade off students of different types, sometimes allowing priorities to determine the choice of a student of one type over a student of another type.

Of course, if priorities always determine choice, then the school cannot maintain any kind of diversity objective. Therefore, the key point is to determine when the school will use priorities and when diversity considerations will prevail. We consider two alternative axioms, both of which roughly say that the number of students of each type determine when priorities are used.

In our axioms, priorities take over once there are "enough" students of the type in question. One axiom states that when there are enough type- $t$ students, then the choice of a type- $t$ student must be explained by the high priority of that student. The second axiom says that when there are not enough type- $t$ students, then the rejection of a type- $t$ student must be explained by the low priority of that student. In other words, a student of a type of which there are few (relative to some diversity objectives) cannot be rejected for diversity reasons.

\footnotetext{
${ }^{3}$ For any vector $\mathbf{x} \in \mathbf{Z}_{+}^{d}$, let $\|\mathbf{x}\|$ be the sum of its coordinates, i.e., $\|\mathbf{x}\| \equiv \sum_{i=1}^{d} \mathbf{x}_{i}$.

${ }^{4}$ Instead of the Euclidean distance, we can work with any $L^{p}$-distance for $p<\infty$.
} 
We proceed to give precise formulations of these two criteria. Our notion of "enough" is called saturation. Our notion of "not enough" is called demanded.

A type $t$ is saturated in a set of students if there is a set of students, with the same number of type- $t$ students, in which not all type- $t$ students are chosen. Saturation says that the number of type- $t$ students has been judged to be enough, so that there are situations with the same number of type- $t$ students in which some type- $t$ students have been rejected.

Now we can introduce an axiom that says that the choice of students of different types must reflect priorities when the chosen student's type is saturated.

SATURATED $\succ-C O M P A T I B I L I T Y:$ Whenever a student whose type is saturated is chosen over another student, the chosen student must have higher priority than the non-chosen student.

Saturated $\succ$-compatibility is one operationalization of the idea that priorities guide choice once there are enough students of one type. It implies within-type $\succ$-compatibility because when the chosen and rejected students have the same type, say type $t$, then type $t$ is saturated in the set.

Our second operationalization is based on the notion of a demanded type. A type $t$ is demanded in a set of students if there is some other set of students with the same number of type- $t$ students in which more type- $t$ students are chosen. That is, a type $t$ is demanded in $S$ if there is $S^{\prime}$ such that $\left|S^{t}\right|=\left|S^{\prime t}\right|$ with $\left|C(S)^{t}\right|<\left|C\left(S^{\prime}\right)^{t}\right|$.

DEMANDED $\succ$-COMPATIBILITY: Whenever a student is chosen over another student whose type is demanded, the chosen student must have higher priority than the non-chosen student.

Now, we describe the first flexible choice rule. A choice rule is generated by reserves for priority $\succ$ if a number of seats is "reserved" for each type. First, for each type $t$, type- $t$ students with the highest priority are chosen until the reserves for type $t$ are filled, or type- $t$ students are exhausted. Next, for the remaining seats, students of the highest priority are chosen until all the seats or students are exhausted. Note that in the second stage, students of all types compete against each other for seats that were either not reserved or reserved but not filled in the first stage.

A choice rule is if a number of seats is "reserved" for each type. First, students of the same type compete for the reserved seats for their type. Then all students compete openly (with students of all types) for the remaining seats including the non-filled reserved seats which happens when the number of students of a given type is less than the number of reserved seats for that type. A student may be turned down in favor of a lower-priority candidate whose quota has not yet been obtained.

More formally, a choice rule $C$ is generated by reserves for priority $\succ$ if there exists a vector $\left(r_{t}\right)_{t \in T} \in \mathbf{Z}_{+}^{d}$ with $\|\mathbf{r}\| \leq q$ such that for any $S \subseteq \mathcal{S}$,

(i) $\left|C(S)^{t}\right| \geq \min \left\{r_{t},\left|S^{t}\right|\right\}$

(ii) if $s \in C(S), s^{\prime} \in S \backslash C(S)$, and $s^{\prime} \succ s$, then it must be the case that $\tau(s) \neq \tau\left(s^{\prime}\right)$ and $\left|C(S)^{\tau(s)}\right| \leq r_{\tau(s)}$; and 
(iii) if $\emptyset \neq S \backslash C(S)$, then $|C(S)|=q$.

If a choice rule is generated by reserves, then it satisfies the desirable property that no student is turned away when there is an empty seat in the school. We call this property acceptance.

ACCEPTANCE: A student is rejected only when all seats are filled.

Acceptance is used in various forms in the literature. It is useful in our characterization of choice rules generated by reserves.

THEOREM 2: A choice rule is generated by reserves for priority $\succ$ if and only if it satisfies gross substitutes, acceptance, and saturated $\succ$-compatibility.

We now turn to our second flexible choice rule. Choice rule $\mathcal{C}$ is generated by quotas for priority $\succ$ if it implements diversity by capping the number of students of each type. More explicitly, the school has a maximum number $r_{t}$ of type- $t$ students that it can admit. Any type- $t$ student in excess of $r_{t}$ will be rejected, regardless of priorities. The school considers all students and chooses the highest-ranked ones conditional on not exceeding any upper bound or the school capacity. In particular, if the sum of the upper bounds is less than the school's capacity, then this model is equivalent to the ideal-point model.

More formally, choice rule $\mathcal{C}$ is generated by quotas for priority $\succ$ if there exists a vector $\left(r_{t}\right)_{t \in T} \in \mathbf{Z}_{+}^{d}$ such that for any $S \subseteq \mathcal{S}$,

(i) $\left|C(S)^{t}\right| \leq r_{t}$;

(ii) if $s \in C(S), s^{\prime} \in S \backslash C(S)$, and $s^{\prime} \succ s$, then it must be the case that $\tau(s) \neq \tau\left(s^{\prime}\right)$ and $\left|C(S)^{\tau\left(s^{\prime}\right)}\right|=r_{\tau\left(s^{\prime}\right)}$; and

(iii) if $s \in S \backslash C(S)$, then either $|C(S)|=q$ or $\left|C(S)^{\tau(s)}\right|=r_{\tau(s)}$.

If a choice rule is generated by quotas, then it can fail the acceptance axiom. Therefore, choice rules generated by reserves may be more favorable than choice rules generated by quotas. Indeed, we provide a welfare comparison for students in the next section (Proposition 1). On the other hand, if a choice rule is generated by quotas then it satisfies the following property.

REJECTION MAXIMALITY (RM): If a type-t student is rejected from a set when there is an empty seat, then the number of type-t students chosen from this set is weakly greater than the corresponding number for any set that does not have more type-t students than this set. $\left(s \in S \backslash C(S)\right.$ and $|C(S)|<q$ imply that, for every $S^{\prime}$ such that $\left|S^{\prime \tau(s)}\right| \leq\left|S^{\tau(s)}\right|$, we have $\left|C(S)^{\tau(s)}\right| \geq\left|C\left(S^{\prime}\right)^{\tau(s)}\right|$.)

Roughly speaking, rejection maximality states that whenever a type- $t$ student is rejected despite the existence of an empty seat, a maximum number of 
type- $t$ students must have been reached. Note that acceptance implies rejection maximality.

THEOREM 3: A choice rule is generated by quotas for priority $\succ$ if and only if it satisfies gross substitutes, rejection maximality, within-type $\succ$-compatibility, and demanded $\succ$-compatibility.

\section{Matching Markets}

We now work out one implication of our results for market-wide outcomes. In a matching market, there are two sets of agents: the set of students and the set of schools. Each student has a strict preference ordering over schools and remaining unmatched whereas each school has a choice rule over groups of students. More formally, a matching market is a tuple $\left\langle\mathcal{C}, \mathcal{S},\left(\succ_{s}\right)_{s \in \mathcal{S}},\left(C_{c}\right)_{c \in \mathcal{C}}\right\rangle$, in which $\mathcal{C}$ is a finite set of schools, $\mathcal{S}$ is a finite set of students, for each $s \in \mathcal{S}$; $\succ_{\mathcal{S}}$ is a strict preference order over $\mathcal{C} \cup\{s\}$ where $\{s\}$ is the outside option for student $s,{ }^{5}$ and for each $c \in \mathcal{C}$; $C_{c}$ is a choice rule over sets of students.

An outcome in this market is a matching that specifies the match for every agent. For matching $\mu$, let $\mu(s)$ be the match of student $s$, which is a school or the outside option, and $\mu(c)$ be the set of students matched with school $c$.

A matching is stable if it satisfies individual rationality and no blocking. First, individual rationality for schools means that no school can be better off by rejecting some of the admitted students, whereas for students it means that each student prefers her assigned school to her outside option. Second, no blocking implies that there exists no coalition of agents who can beneficially rematch among themselves. This is the standard definition of stability used in many-to-one matching problems (Roth and Sotomayor 1990).

The student-optimal stable mechanism (SOSM) is defined through the algorithm of Gale and Shapley (1962). When schools' choices satisfy gross substitutes, SOSM produces the best stable matching for students. ${ }^{6}$ SOSM has been implemented in many matching markets, including several school districts and markets for entrylevel professional jobs (Roth 2008; Pathak and Sönmez 2013).

Suppose that schools' choice rules are generated using the quota model. Then there can be situations in which a student is turned away from a school even though there is an empty seat. We can avoid this by using reserves instead of quotas. In the next result, we study the effects of this change on students' welfare, and show that each student weakly prefers reserves to quotas when SOSM is in place.

PROPOSITION 1: Suppose that SOSM is used in a matching market. Then each student weakly prefers the outcome when schools' choice rules are generated by reserves to the outcome when schools' choice rules are generated by quotas, if each school uses its vector of reserves as quotas.

\footnotetext{
${ }^{5}$ The outside option for student $s$ can be going to a private school or being homeschooled.

${ }^{6}$ In addition to GS, another axiom called irrelevance of rejected students is also needed. Irrelevance of rejected students is implied by our other axioms. See Appendix A for the definition.
} 
Proposition 1 follows from a more general result that we show in the online Appendix (Theorem E.1).

\section{Appendix A: Proofs of Theorems 1-3}

All our results are based on mapping a choice rule into a function $f:\left\{\mathbf{x} \in \mathbf{Z}_{+}^{d}: 0 \leq \mathbf{x} \leq \xi(\mathcal{S})\right\} \rightarrow \mathbf{Z}_{+}^{d}$, mapping distributions into distributions. The axioms have properties of such functions as counterparts. We begin with some preliminary results on functions that map distributions into distributions.

We say that $f$ is monotone increasing if $\mathbf{y} \leq \mathbf{x}$ implies that $f(\mathbf{y}) \leq f(\mathbf{x}) ; f$ is within budget if $f(\mathbf{x}) \in B(\mathbf{x})$; that $f$ satisfies gross substitutes if

$$
\mathbf{y} \leq \mathbf{x} \Rightarrow f(\mathbf{x}) \wedge \mathbf{y} \leq f(\mathbf{y})
$$

A function $f$ is generated by an ideal point if there is $\mathbf{z}^{*} \in \mathbf{Z}_{+}^{d}$ such that $\left\|\mathbf{z}^{*}\right\| \leq q$, and $f(\mathbf{x})$ minimizes the Euclidean distance to $\mathbf{z}^{*}$ among the vectors in $B(\mathbf{x})$.

We shall first introduce some simple lemmas related to functions on $\mathbf{Z}_{+}^{d}$. For any $\mathbf{x}, \mathbf{y} \in \mathbf{Z}_{+}^{d}$, let $\mathbf{x} \wedge \mathbf{y} \equiv\left(\min \left\{\mathbf{x}_{1}, \mathbf{y}_{1}\right\}, \ldots, \min \left\{\mathbf{x}_{d}, \mathbf{y}_{d}\right\}\right)$ be the infimum of $\mathbf{x}$ and $\mathbf{y}$.

LEMMA 1: Let $\mathbf{z}^{*} \in \mathbf{Z}_{+}^{d}$ satisfy $\left\|\mathbf{z}^{*}\right\| \leq q$. Then $\mathbf{x} \wedge \mathbf{z}^{*}$ is the unique minimizer of the Euclidean distance to $\mathbf{z}^{*}$ among the vectors in $B(\mathbf{x})$.

\section{PROOF:}

First note that $\mathbf{x} \wedge \mathbf{z}^{*} \in B(\mathbf{x})$. The distance from $\mathbf{z}$ to $\mathbf{z}^{*}$ is minimized if $\sum_{t}\left(\mathbf{z}_{t}-\mathbf{z}_{t}^{*}\right)^{2}$ is minimized. But for each $t,\left(\mathbf{z}_{t}-\mathbf{z}_{t}^{*}\right)^{2}$ is minimized by setting $\mathbf{z}_{t}=\min \left\{\mathbf{x}_{t}, \mathbf{z}_{t}^{*}\right\}:$ when $\min \left\{\mathbf{x}_{t}, \mathbf{z}_{t}^{*}\right\}=\mathbf{z}_{t}^{*}$ this is trivial, and when $\min \left\{\mathbf{x}_{t}, \mathbf{z}_{t}^{*}\right\}=\mathbf{x}_{t}$ then there is no $\mathbf{z} \in B(\mathbf{x})$ with $\mathbf{z}_{t}>\mathbf{x}_{t}$. Since $\mathbf{z}_{t}=\min \left\{\mathbf{x}_{t}, \mathbf{z}_{t}^{*}\right\}$ for every $t$, we get $\mathbf{z}=\mathbf{x} \wedge \mathbf{z}^{*}$.

LEMMA 2: Function $f$ is generated by an ideal point if and only if it is monotone increasing, within budget, and satisfies gross substitutes.

PROOF:

We first show that if $f$ is generated by an ideal point $\mathbf{z}^{*}$, then it is monotone increasing, within budget and it satisfies gross substitutes. Suppose that the ideal point is $\mathbf{z}^{*}$. By Lemma $1, f(\mathbf{x})=\mathbf{x} \wedge \mathbf{z}^{*}$. Then $f(\mathbf{x}) \leq \mathbf{x}$ and $\|f(\mathbf{x})\| \leq$ $\left\|\mathbf{z}^{*}\right\| \leq q$, so $f$ is within budget. Next we show monotonicity: $\mathbf{y} \leq \mathbf{x} \Rightarrow \mathbf{y} \wedge$ $\mathbf{z}^{*} \leq \mathbf{x} \wedge \mathbf{z}^{*} \Rightarrow f(\mathbf{y}) \leq f(\mathbf{x})$. Last we show gross substitutes. Let $\mathbf{y} \leq \mathbf{x}$. Then, $f(\mathbf{x}) \wedge \mathbf{y}=\left(\mathbf{x} \wedge \mathbf{z}^{*}\right) \wedge \mathbf{y}=(\mathbf{x} \wedge \mathbf{y}) \wedge \mathbf{z}^{*}=\mathbf{y} \wedge \mathbf{z}^{*}=f(\mathbf{y})$

We now turn to proving that the axioms are sufficient for generation by an ideal point. We suppose that $f$ is a function satisfying monotonicity, gross substitutes, and it is within budget.

Note if $\mathbf{y} \leq \mathbf{x}$ then the monotonicity of $f$, and that $f(\mathbf{y}) \leq \mathbf{y}$ implies that $f(\mathbf{y}) \leq \mathbf{y} \wedge f(\mathbf{x})$. Thus the GS axiom becomes

$$
\mathbf{y} \leq \mathbf{x} \Rightarrow f(\mathbf{x}) \wedge \mathbf{y}=f(\mathbf{y})
$$


Let $\hat{\mathbf{x}}$ be such that $\hat{\mathbf{x}}_{t}>q$ for all $t$. For arbitrary $\mathbf{y}$, we shall prove that $f(\mathbf{y})=\mathbf{y} \wedge f(\hat{\mathbf{x}})$. Note that $\hat{\mathbf{x}} \wedge \mathbf{y} \leq \hat{\mathbf{x}}$, monotonicity, and gross substitutes, imply (using equation (A1)) that $f(\hat{\mathbf{x}} \wedge \mathbf{y})=(\hat{\mathbf{x}} \wedge \mathbf{y}) \wedge f(\hat{\mathbf{x}})=(\hat{\mathbf{x}} \wedge f(\hat{\mathbf{x}})) \wedge \mathbf{y}$ $=f(\hat{\mathbf{x}}) \wedge \mathbf{y}$. Similarly, $\hat{\mathbf{x}} \wedge \mathbf{y} \leq \mathbf{y}$ gives us that $f(\hat{\mathbf{x}} \wedge \mathbf{y})=(\hat{\mathbf{x}} \wedge \mathbf{y}) \wedge f(\mathbf{y})$ $=(\mathbf{y} \wedge f(\mathbf{y})) \wedge \hat{\mathbf{x}}=f(\mathbf{y}) \wedge \hat{\mathbf{x}}$. Thus, $f(\hat{\mathbf{x}}) \wedge \mathbf{y}=f(\mathbf{y}) \wedge \hat{\mathbf{x}}$. But since $f(\mathbf{y})_{t} \leq q$ for all $t$, we have, $f(\mathbf{y}) \wedge \hat{\mathbf{x}}=f(\mathbf{y})$. Therefore, $f(\mathbf{y})=f(\hat{\mathbf{x}}) \wedge \mathbf{y}$. By Lemma $1, f(\mathbf{y})$ minimizes the distance to $f(\hat{\mathbf{x}})$ in $B(\mathbf{y})$. Therefore, $f$ is generated by ideal point $f(\hat{\mathbf{x}})$.

We also need the following axiom in what follows.

IRRELEVANCE OF REJECTED STUDENTS (IRS): Choice rule C satisfies IRS if $C\left(S^{\prime}\right) \subseteq S \subseteq S^{\prime}$ implies that $C(S)=C\left(S^{\prime}\right)$.

The following simple result follows from well-known arguments in choice theory. Its proof is omitted.

LEMMA 3: If C satisfies GS and acceptance, then it also satisfies IRS.

\section{PROOF OF THEOREM 1:}

Suppose that $C$ satisfies the axioms. We argue that it is generated by an ideal point for priority $\succ$.

Define $f:\left\{\mathbf{x} \in \mathbf{Z}_{+}^{d}: 0 \leq \mathbf{x} \leq \xi(\mathcal{S})\right\} \rightarrow \mathbf{Z}_{+}^{d}$ as follows. For any $\mathbf{x} \leq \xi(\mathcal{S})$, consider $S$ such that $\mathbf{x}=\xi(S)$ and let $f(\mathbf{x})=\xi(C(S))$. By monotonicity, $\xi(S)$ $=\xi\left(S^{\prime}\right)$ implies $\xi(C(S))=\xi\left(C\left(S^{\prime}\right)\right)$; hence the particular choice of $S$ does not matter, therefore $f$ is well defined. Moreover, when $\mathbf{y} \leq \mathbf{x}$ we have $f(\mathbf{y}) \leq f(\mathbf{x})$, again by monotonicity. So $f$ is monotone increasing. In addition, $f(\mathbf{x}) \leq \mathbf{x}$ and $\|f(\mathbf{x})\| \leq q$; so $f$ is within budget.

To show that $f$ satisfies GS, let $\xi(\mathcal{S}) \geq \mathbf{x} \geq \mathbf{y}$ and $S^{\prime} \subseteq \mathcal{S}$ be such that $\xi\left(S^{\prime}\right)=\mathbf{x}$. Construct $S$ with $\xi(S)=\mathbf{y}$ as follows. For any $t$, if $\mathbf{y}_{t} \geq \xi\left(C\left(S^{\prime}\right)\right)_{t}$, then $S^{t} \supseteq C\left(S^{\prime}\right)^{t}$. However, if $\mathbf{y}_{t}<\xi\left(C\left(S^{\prime}\right)\right)_{t}$, then $S^{t} \subseteq C\left(S^{\prime}\right)^{t}$. In the former case $C(S)^{t} \supseteq C\left(S^{\prime}\right)^{t}$, and in the latter case $C(S)^{t}=S^{t}$ by GS. In both cases, $\xi(C(S))_{t} \geq \min \left\{\xi(S)_{t}, \xi\left(C\left(S^{\prime}\right)\right)_{t}\right\}$ for any $t$, which implies $f(\mathbf{y}) \geq f(\mathbf{x}) \wedge \mathbf{y}$.

By Lemma $2, f$ is generated by the ideal point $\mathbf{z}^{*} \equiv f(\hat{\mathbf{x}})$ where $\hat{\mathbf{x}}$ is such that $\hat{\mathbf{x}}_{t}>q$ for all $t$. Since $f\left(\mathbf{z}^{*}\right)=\mathbf{z}^{*}$, we have that $\left\|\mathbf{z}^{*}\right\| \leq q$. Therefore, $C$ is generated by $\mathbf{z}^{*}$ for $\succ$ because (i) $f$ is generated by the ideal point $\mathbf{z}^{*}$ implies $\xi(C(S))$ is the closest vector to $\mathbf{z}^{*}$ in $B(\xi(S))$ for every $S$, and (ii) within-type $\succ$-compatibility implies that any type- $t$ student in $C(S)$ has a higher priority than any student in $S \backslash C(S)$ for every $S$.

Conversely, let $C$ be generated an ideal point for priority $\succ$. It is immediate that $C$ satisfies within-type $\succ$-compatibility.

Define $f$ from $C$ as above: $f$ is well defined because for any $S$ and $S^{\prime}$ such that $\xi(S)=\xi\left(S^{\prime}\right)=\mathbf{x}, \xi(C(S))$ is the closest vector to $\mathbf{z}^{*}$ among those in $B(\mathbf{x})$ and $\xi\left(C\left(S^{\prime}\right)\right)$ is the closest vector to $\mathbf{z}^{*}$ among those in $B(\mathbf{x})$. Therefore, $\xi(C(S))$ $=\xi\left(C\left(S^{\prime}\right)\right)$ by Lemma 1 .

To show that $C$ satisfies monotonicity, let $\mathbf{y}=\xi(S)$ and $\mathbf{x}=\xi\left(S^{\prime}\right)$ such that $\mathbf{y} \leq \mathbf{x}$. By Lemma $1, f(\mathbf{x})=\mathbf{x} \wedge \mathbf{z}^{*}$ and $f(\mathbf{y})=\mathbf{y} \wedge \mathbf{z}^{*}$. Then, $f(\mathbf{y})$ $=\mathbf{y} \wedge \mathbf{z}^{*} \leq \mathbf{x} \wedge \mathbf{z}^{*}=f(\mathbf{x})$, and, therefore, $\xi(C(S)) \leq \xi\left(C\left(S^{\prime}\right)\right)$. 
To see that $C$ satisfies GS, let $s \in S \subseteq S^{\prime}, \tau(s)=t, \xi(S)=\mathbf{y}$ and $\xi\left(S^{\prime}\right)=\mathbf{x}$. As we have shown above, $f(\mathbf{x})=\mathbf{x} \wedge \mathbf{z}^{*}$ and $f(\mathbf{y})=\mathbf{y} \wedge \mathbf{z}^{*}$. If $f(\mathbf{y})_{t} \geq f(\mathbf{x})_{t}$, then more type $t$ students are chosen in $S$ compared to $S^{\prime}$. Since $s \in C\left(S^{\prime}\right)$, and $C$ is generated by an ideal point, we derive that $s \in C(S)$. On the other hand, if $f(\mathbf{y})_{t}<$ $f(\mathbf{x})_{t}$, then $f(\mathbf{y})_{t}<\mathbf{z}_{t}^{*}$ since $f(\mathbf{x})_{t}=\left(\mathbf{x} \wedge \mathbf{z}^{*}\right)_{t} \leq \mathbf{z}_{t}^{*}$. Since $f(\mathbf{y})_{t}=\left(\mathbf{y} \wedge \mathbf{z}^{*}\right)_{t}$, we derive that $f(\mathbf{y})_{t}=\mathbf{y}_{t}$. That means that all type $t$ students are chosen from $S$; so $s \in C(S)$.

\section{PROOF OF THEOREM 2:}

For any $\mathbf{x} \leq \xi(\mathcal{S})$, let $F(\mathbf{x}) \equiv\{\xi(C(S)): \xi(S)=\mathbf{x}\}$ and

$$
\hat{f}(\mathbf{x})=\bigwedge_{f(\mathbf{x}) \in F(\mathbf{x})} f(\mathbf{x})
$$

The proof of the theorem requires the following lemma.

LEMMA 4: Let C satisfy GS. If $\mathbf{y} \in \mathbf{Z}_{+}^{d}$ is such that $\hat{f}(\mathbf{y})_{t}<\mathbf{y}_{t}$ then $\hat{f}\left(\mathbf{y}+e_{t^{\prime}}\right)_{t}<$ $\mathbf{y}_{t}+1_{t=t^{\prime}}$.

PROOF:

Let $\mathbf{y}$ and $t$ be as in the statement of the lemma. Let $S$ be such that $\xi(S)=\mathbf{y}$ and $\xi(C(S))_{t}<\xi(S)_{t}=\mathbf{y}_{t}$. Such a set $S$ exists because $\hat{f}(\mathbf{y})_{t}<\mathbf{y}_{t}$. Let $s^{\prime} \notin S$ be an arbitrary student with $\tau\left(s^{\prime}\right)=t^{\prime}$. Note that

$$
\emptyset \neq S^{t} \backslash C(S)^{t} \subseteq\left(S \cup\left\{s^{\prime}\right\}\right)^{t} \backslash C\left(S \cup\left\{s^{\prime}\right\}\right)^{t},
$$

as $C$ satisfies GS. Then we cannot have $\xi\left(C\left(S \cup\left\{s^{\prime}\right\}\right)\right)_{t}=\mathbf{y}_{t}+1_{t=t^{\prime}}$ because that would imply $\left(S \cup\left\{s^{\prime}\right\}\right)^{t} \backslash C\left(S \cup\left\{s^{\prime}\right\}\right)^{t}=\emptyset$. Then

$$
\mathbf{y}_{t}+1_{t=t^{\prime}}>\xi\left(C\left(S \cup\left\{s^{\prime}\right\}\right)\right)_{t} \geq \hat{f}\left(\mathbf{y}+e_{t^{\prime}}\right)_{t}
$$

Suppose that $C$ satisfies the axioms. Construct $r$ as follows. Let $\overline{\mathbf{x}}=\xi(\mathcal{S})$. Lemma 4 implies that if $\hat{f}\left(\mathbf{y}_{t}, \overline{\mathbf{x}}_{-t}\right)_{t}<\mathbf{y}_{t}$ then $\hat{f}\left(y_{t}^{\prime}, \overline{\mathbf{x}}_{-t}\right)_{t}<y_{t}^{\prime}$ for all $y_{t}^{\prime}>y_{t}$. Then there is $r_{t} \in N$ such that $\mathbf{y}_{t}>r_{t}$ if and only if $\hat{f}\left(\mathbf{y}_{t}, \overline{\mathbf{x}}_{-t}\right)<\mathbf{y}_{t}$. This uses the assumption on the cardinality of $\mathcal{S}^{t}$ that $\hat{f}(\mathbf{y})_{t}<\mathbf{y}_{t}$ if $\mathbf{y}_{t}$ is large enough. Note that we may have $r_{t}=0$.

First, we prove if $S \subseteq \mathcal{S}$ with $\left|S^{t}\right| \leq \mathbf{r}_{t}$ then $S^{t}=C(S)^{t}$. Observe that, for any $\mathbf{x}$ and $t, \hat{f}\left(\mathbf{r}_{t}, \mathbf{x}_{-t}\right)=\mathbf{r}_{t}$. To see this, note that if there is $\mathbf{x}$ and $t$ such that $\hat{f}\left(\mathbf{r}_{t}, \mathbf{x}_{-t}\right)<\mathbf{r}_{t}$ then Lemma 4 would imply that $\hat{f}\left(\mathbf{r}_{t}, \overline{\mathbf{x}}_{-t}\right)<\mathbf{r}_{t}$, in contradiction with the definition of $r$. In fact, we can say more: for any $\mathbf{x}, t$, and $\mathbf{y}_{t}$, if $\mathbf{y}_{t} \leq \mathbf{r}_{t}$ then $\hat{f}\left(\mathbf{r}_{t}, \mathbf{x}_{-t}\right)=\mathbf{r}_{t}$ and Lemma 4 imply that $\hat{f}\left(\mathbf{y}_{t}, \mathbf{x}_{-t}\right)=\mathbf{y}_{t}$. Therefore, letting $S \subseteq \mathcal{S}$ with $\left|S^{t}\right| \leq \mathbf{r}_{t}$ we have that

$$
\left|C(S)^{t}\right| \geq \hat{f}(\mathbf{y})_{t}=\mathbf{y}_{t}
$$


where $\mathbf{y}=\xi(S)$. Since $\mathbf{y}_{t}=\left|S^{t}\right| \geq\left|C(S)^{t}\right|$ we have that $S^{t}=C(S)^{t}$.

Second, we prove that, if $\left|S^{t}\right|>\mathbf{r}_{t}$, then $\left|C(S)^{t}\right| \geq \mathbf{r}_{t}$. Let $\tilde{S}=C(S)$. Assume, toward a contradiction, that $\left|\tilde{S}^{t}\right|<\mathbf{r}_{t}$. Let $S^{\prime}=\tilde{S} \cup S^{\prime \prime}$, where $S^{\prime \prime} \subseteq S^{t} \backslash \tilde{S}^{t}$ is such that $\left|S^{\prime t}\right|=\mathbf{r}_{t}$. By Lemma 3, $C$ satisfies IRS, so $C\left(S^{\prime}\right)=C(S)$. Thus,

$$
\hat{f}\left(\xi\left(S^{\prime}\right)\right)_{t} \leq\left|C\left(S^{\prime}\right)^{t}\right|=\left|C(S)^{t}\right|<\mathbf{r}_{t}
$$

Since $\xi\left(S^{\prime}\right)_{t}=\left|S^{\prime t}\right|=\mathbf{r}_{t}$, we obtain a contradiction with the definition of $\mathbf{r}_{t}$ above.

Third, suppose that $s \in C(S), s^{\prime} \in S \backslash C(S)$ and $s^{\prime} \succ s$. Since $s^{\prime} \in S \backslash C(S)$, $\tau\left(s^{\prime}\right)$ is saturated in $S$. If $\tau(s)=\tau\left(s^{\prime}\right)$, we get a contradiction with saturated $\succ$-compatibility and $s^{\prime} \succ s$. Therefore, $\tau(s) \neq \tau\left(s^{\prime}\right)$. If $\left|C(S)^{\tau(s)}\right|>\mathbf{r}_{\tau(s)}$, then by the construction of $\mathbf{r}_{t}$ type $\tau(s)$ must be saturated in $S$. But this contradicts saturated $\succ$-compatibility and $s^{\prime} \succ s$, so $\mid C(S)^{\tau(s) \mid} \leq \mathbf{r}_{\tau(s)}$.

It remains to show that if $C$ is generated by reserves for priority $\succ$, then it satisfies the axioms. It is immediate that it satisfies acceptance and saturated $\succ$-compatibility.

To see that it satisfies GS, let $S \subseteq S^{\prime}$ and $s \in S \backslash C(S)$. Then $\left|S^{\tau(s)}\right|>\mathbf{r}_{\tau(s)}$; so $\left|S^{\prime \tau(s)}\right|>\mathbf{r}_{\tau(s)}$. Moreover, $s \in S \backslash C(S)$ implies that there are $\mathbf{r}_{\tau(s)}$ students in $S^{\tau(s)}$ ranked above $s$. Let $C^{(1)}(S)$ be the set of students that are accepted in the first step (on reserved seats), $S^{*}$ be the set of students that are considered in the second step (on open seats), and $q^{*}$ be the number of remaining seats to be allocated in the second step. Again, $s \in S \backslash C(S)$ implies that there are $q^{*}$ students ranked above $s$ in $S^{*}$. Consider the construction of $C\left(S^{\prime}\right): s$ cannot be admitted in the first step since $S^{\prime \tau(s)} \supseteq S^{\tau(s)}$ and that there are at least $r_{\tau(s)}$ students ranked above $s$ in $S^{\tau(s)}$. Furthermore, in the second step of the new procedure, there are more higher ranked students of each type compared to $S^{*}$, so $s$ can also not be admitted in the second step since there are at most $q^{*}$ seats left. Therefore, $s \in S^{\prime} \backslash C\left(S^{\prime}\right)$.

\section{PROOF OF THEOREM 3:}

Suppose that $C$ satisfies the axioms and let $\mathbf{r}_{t} \equiv \max _{S \in \mathcal{S}}\left|C(S)^{t}\right|$.

LEMMA 5: Suppose $S^{\prime} \subseteq \mathcal{S}^{t}$. If $C\left(S^{\prime}\right)<\min \left\{q,\left|S^{\prime}\right|\right\}$ then $C\left(S^{\prime}\right)=\mathbf{r}_{t}$.

\section{PROOF:}

Since $\mathbf{r}_{t}=\max _{S \in \mathcal{S}}\left|C(S)^{t}\right|$, there exists a set $\bar{S}$ such that $\left|C(\bar{S})^{t}\right|=\mathbf{r}_{t}$. By GS, we can choose $\bar{S}$ such that $\bar{S} \subseteq \mathcal{S}^{t}$ and $\bar{S}=C(\bar{S})$ (simply choose $C(\bar{S})^{t}$ to be the set in question). Now let $S^{\prime}$ be a set of students as in the statement of the lemma. Suppose toward a contradiction that $C\left(S^{\prime}\right)<\mathbf{r}_{t}$.

Note that $C\left(S^{\prime}\right)<\min \left\{q,\left|S^{\prime}\right|, \mathbf{r}_{t}\right\}$ and $|C(\bar{S})|=\mathbf{r}_{t}$. So RM implies that $|\bar{S}|>\left|S^{\prime}\right|$.

Let $P \subseteq \bar{S}$ be a set of cardinality $\left|S^{\prime}\right|$. By GS, $\bar{S}=C(\bar{S})$ implies that $P=C(P)$, so $|C(P)|=\left|S^{\prime}\right|>C\left(S^{\prime}\right)$, a contradiction to RM.

To show that $C$ is generated by quotas for priority $\succ$ we need three things. First, $\left|C(S)^{t}\right| \leq \mathbf{r}_{t}$ for every $S \subseteq \mathcal{S}$. This is immediate by construction of $\mathbf{r}_{t}$. Second, 
we show that if $s \in C(S), s^{\prime} \in S \backslash C(S)$, and $s^{\prime} \succ s$, then it must be the case that $\tau(s) \neq \tau\left(s^{\prime}\right)$ and $\left|C(S)^{\tau\left(s^{\prime}\right)}\right|=\mathbf{r}_{\tau\left(s^{\prime}\right)}$. If $\tau(s)=\tau\left(s^{\prime}\right)$, we get a contradiction to within-type $\succ$-compatibility. If $\left|C(S)^{\tau\left(s^{\prime}\right)}\right| \neq \mathbf{r}_{\tau\left(s^{\prime}\right)}$, then $\left|C(S)^{\tau\left(s^{\prime}\right)}\right|<\mathbf{r}_{\tau\left(s^{\prime}\right)}$ by construction of $r$. We shall prove that $\tau\left(s^{\prime}\right)$ is demanded in $S$, which will yield the desired contradiction to demanded $\succ$-compatibility. Let $S^{\prime} \equiv S^{\tau\left(s^{\prime}\right)}$. We consider three cases.

- First, if $\left|C\left(S^{\prime}\right)\right|=q$ then $\left|C(S)^{\tau\left(s^{\prime}\right)}\right|<\left|C\left(S^{\prime}\right)^{\tau\left(s^{\prime}\right)}\right|$ (as $s \in C(S)$ and $\tau(s)$ $\left.\neq \tau\left(s^{\prime}\right)\right)$, so $\tau\left(s^{\prime}\right)$ is demanded in $S$.

- Second, if $\left|C\left(S^{\prime}\right)\right|<q$ and $\left|C\left(S^{\prime}\right)\right|<\left|S^{\prime}\right|$, then, by Lemma 5, $\left|C\left(S^{\prime}\right)\right|=\mathbf{r}_{\tau\left(s^{\prime}\right)}$, so $\left|C\left(S^{\prime}\right)\right|>\left|C(S)^{\tau\left(s^{\prime}\right)}\right|$. Hence $\tau\left(s^{\prime}\right)$ is demanded in $S$.

- Third, consider the case when $\left|C\left(S^{\prime}\right)\right|<q$, and $\left|C\left(S^{\prime}\right)\right|=\left|S^{\prime}\right|$. Then $\left|C\left(S^{\prime}\right)\right|>$ $\left|C(S)^{\tau\left(s^{\prime}\right)}\right|$, as $s^{\prime} \in S^{\tau\left(s^{\prime}\right)} \backslash C(S)^{\tau\left(s^{\prime}\right)}$. Thus $\tau\left(s^{\prime}\right)$ is demanded in $S$.

Finally, we need to show that if $s \in S \backslash C(S)$, then either $|C(S)|=q$ or $\left|C(S)^{\tau(s)}\right|=\mathbf{r}_{\tau(s)}$. Suppose that $|C(S)|<q$. Let $S^{\prime} \equiv S^{\tau(s)}$. By RM, $\left|C(S)^{\tau(s)}\right|$ $\geq\left|C\left(S^{\prime}\right)\right|$, so $\left|C\left(S^{\prime}\right)\right|<q$ since $|C(S)|<q$. Similarly $\left|C\left(S^{\prime}\right)\right|<\left|S^{\prime}\right|$, because otherwise $\left|C(S)^{\tau(s)}\right| \geq\left|C\left(S^{\prime}\right)\right|$ would imply $C(S)^{\tau(s)}=S^{\prime}$; a contradiction since $s \in S \backslash C(S)$. We have established $\left|C\left(S^{\prime}\right)\right|<\min \left\{q,\left|S^{\prime}\right|\right\}$, so by Lemma 5 we get $\left|C\left(S^{\prime}\right)\right|=\mathbf{r}_{\tau(s)}$.

To finish the proof, suppose that $C$ is generated by quotas for $\succ$. Then it is easy to see that $C$ satisfies RM, within-type $\succ$-compatibility and demanded $\succ$-compatibility. We show that it also satisfies GS. Suppose that $s \in S \subseteq S^{\prime}$ and $s \in C\left(S^{\prime}\right)$. For each type $t$, let $S\left(t ; \mathbf{r}_{t}\right) \subseteq S^{t}$ be the $\mathbf{r}_{t}$ highest ranked type $t$ students in $S$ (if $\left|S^{t}\right|$ $\leq \mathbf{r}_{t}$ then $\left.S\left(t ; \mathbf{r}_{t}\right)=S^{t}\right)$. Define $S^{\prime}\left(t ; \mathbf{r}_{t}\right)$ analogously. Since $s \in C\left(S^{\prime}\right)$, we have $s \in S^{\prime}\left(\tau(s), \mathbf{r}_{\tau(s)}\right)$ and the ranking of $s$ in $\cup_{t} S^{\prime}\left(t ; \mathbf{r}_{t}\right)$ is no more than $q$. Since $S \subseteq S^{\prime}$, the preceding statements also hold for $S$ instead of $S^{\prime}$, which implies that $s \in C(S)$.

\section{REFERENCES}

Abdulkadiroğlu, Atila, Parag A. Pathak, Alvin E. Roth, and Tayfun Sönmez. 2005. "The Boston Public School Match.” American Economic Review 95 (2): 368-71.

Abdulkadiroğlu, Atila, and Tayfun Sönmez. 2003. "School Choice: A Mechanism Design Approach." American Economic Review 93 (3): 729-47.

-Alkan, Ahmet, and David Gale. 2003. “Stable Schedule Matching under Revealed Preference.” Journal of Economic Theory 112 (2): 289-306.

Alves, Michael J., and Charles V. Willie. 1987. "Controlled Choice Assignments: A New and More Effective Approach to School Desegregation.” Urban Review 19 (2): 67-88.

Aygün, Orhan, and Inacio Bo. 2013. "College Admission with Multidimensional Reserves: The Brazilian Affirmative Action Case.” www.inaciobo.com/AygunBo2014.pdf (accessed June 1, 2015).

Echenique, Federico, and M. Bumin Yenmez. 2007. "A Solution to Matching with Preferences over Colleagues." Games and Economic Behavior 59 (1): 46-71.

-Ehlers, Lars, Isa E. Hafalir, M. Bumin Yenmez, and Muhammed A. Yildirim. 2014. "School Choice with Controlled Choice Constraints: Hard Bounds versus Soft Bounds." Journal of Economic Theory 153: 648-83.

Fiske, Edward B. 2002. Controlled Choice in Cambridge, Massachusetts. New York: Century Foundation Press.

Gale, David, and Lloyd S. Shapley. 1962. “College Admissions and the Stability of Marriage." American Mathematical Monthly 69 (1): 9-15. 
Hafalir, Isa E., M. Bumin Yenmez, and Muhammed A. Yildirim. 2013. "Effective Affirmative Action in School Choice." Theoretical Economics 8 (2): 325-63.

-Hatfield, John William, and Paul R. Milgrom. 2005. "Matching with Contracts." American Economic Review 95 (4): 913-35.

Kahlenberg, Richard D. 2002. All Together Now: Creating Middle-Class Schools through Public School Choice. Washington, DC: Brookings Institution Press.

Kelso, Alexander S., Jr., and Vincent P. Crawford. 1982. "Job Matching, Coalition Formation, and Gross Substitutes.” Econometrica 50 (6): 1483-1504.

Kojima, Fuhito. 2012. "School Choice: Impossibilities for Affirmative Action." Games and Economic Behavior 75 (2): 685-93.

Kominers, Scott Duke, and Tayfun Sönmez. 2012. "Designing for Diversity: Matching with Slot-Specific Priorities." http://fmwww.bc.edu/EC-P/wp806.pdf (accessed June 1, 2015).

Pathak, Parag A., and Tayfun Sönmez. 2013. "School Admissions Reform in Chicago and England: Comparing Mechanisms by Their Vulnerability to Manipulation.” American Economic Review 103 (1): 80-106.

Pycia, Marek. 2012. "Stability and Preference Alignment in Matching and Coalition Formation." Econometrica 80 (1): 323-62.

Roth, Alvin E. 2008. "Deferred Acceptance Algorithms: History, Theory, Practice, and Open Questions." International Journal of Game Theory 36 (3-4): 537-69.

Roth, Alvin E., and Marilda A. Oliveira Sotomayor. 1990. Two-Sided Matching: A Study in Game-Theoretic Modeling and Analysis. Cambridge, UK: Cambridge University Press.

-Westkamp, Alexander. 2013. "An Analysis of the German University Admissions System.” Economic Theory 53 (3): 561-89. 